\title{
DRUG INDUCED DERMATOLOGICAL REACTION OF THE 100 MOST COMMONLY PRESCRIBED MEDICATIONS IN UK HOSPITALS
}

\author{
MOHAMMED AL-ABADIE ${ }^{1}$, FARIS OUMEISH ${ }^{1}$, MOHAMMED AL-RUBAYE ${ }^{1}$, DINA AL-ABADIE ${ }^{2}$, PATRICK ANTHONY \\ BALL $^{3}$, HANA MORRISSEY ${ }^{3}$
}

${ }^{1}$ Department of Dermatology Royal Wolverhampton, NHS Trust, Wolverhampton, United Kingdom, ${ }^{2}$ Department of Health Promotion and Public Health, University of West London, London, United Kingdom, ${ }^{3}$ School of Pharmacy, University of Wolverhampton, Wolverhampton, United Kingdom

Email: mohammed.abadie@nhs.net

Received: 12 May 2019, Revised and Accepted: 15 Jul 2019

\begin{abstract}
Objective: It is commonly reported that medicines have side effects related to dermatological practice. However, it is extremely difficult to establish how commonly, or rarely skin-related medication side effects occur. Common dermatological side effects include rash, pruritus, and
\end{abstract} photosensitivity.

Objective: To demonstrate the dermatological side-effects of the most commonly prescribed medications in the United Kingdom.

Methods: This paper discusses dermatological side-effects of the commonly prescribed medications, including uncommon or rare manifestations such as angioedema and Stevens - Johnson syndrome (SJS). The list used for the most frequently prescribed drugs in the United Kingdom was created by nurses. This list was compared to the British National Formulary to demonstrate the reported frequency of occurrence of dermatological side-effects or complications.

Conclusion: The top 100 prescribed medication cause a number of dermatological side effects that need to be considered when they are prescribed to patients who have pre-existing skin conditions. Additionally, when confronted with a common dermatological problem in any patient, clinicians should always consider the possibility of a drug adverse reaction.

Keywords: Drug side effects, Drug-induced stevens-johnson syndrome, Drug-induced licheniform eruptions, Photosensitizing effect, Exanthematous pustulosis

(c) 2019 The Authors. Published by Innovare Academic Sciences Pvt Ltd. This is an open-access article under the CC BY license (http://creativecommons.org/licenses/by/4.0/) DOI: http://dx.doi.org/10.22159/ijcpr.2019v11i5.35703

\section{INTRODUCTION}

Dermatological adverse reactions are thought to be common, but the true incidence remains speculative because the reactions resemble commonly occurring skin conditions and the true cause may, therefore, be missed. Examples would include excessive sensitivity to UV, mainly the ultraviolet spectrum B (UVB, 280-320 nm) broadband (UVB-BB, 380-320 nm) present in normal sunlight but also used in the treatment of psoriasis [1]. Common medications known to cause this reaction include: griseofulvin, sulphonamides, sulphonylureas, thiazide diuretics, amiodarone, nalidixic acid, tetracyclines and chlorpromazine [2]. Skin presentation may appear after weeks or months and appear eczematous, lichenoid, urticarial, bullous or may appear as a purpuric eruption.

Some medications may cause skin tone changes; which may be hyper-or hypopigmentation. It may be widespread or localised e. g., metabolic disposition in the dermis such as; skin stain after using silver dressings or enhanced melanin production with or without an increase in melanocytes as occurs with antimalarials, amiodarone, NSAIDs, and bleomycin sulphate [3].

Others may cause nail changes such as chemotherapy (white, depression, and separation from the nail-bed, beau's lines or interruption of normal growth), minocycline (blue-black stain) or potassium permanganate (brown stain) [4]. Hair changes can also occur; total loss of hair (alopecia) where the drug interferes in the anagen (hair growth) phase, partial or complete temporary alopecia e. g. cytotoxic drugs (reversible after ceasing treatment, dose-dependent, start after short period of drug commencement), delay in hair growth (telogen phase or the shedding cycle faster than growth cycle), retinoid therapy and danazol therapy (Hirsutism/Hypertrichosis in women or change in hair pattern in men [5].
Some medications, as part of their activity, cause changes to the skin's immune system and may promote skin malignancy; e. g., ciclosporin, mycophenolate mofetil, tacrolimus [6]. Drug-induced exanthems is a widespread rash, erythema (redness, $<90 \%$ of body surface area), morbilliform or maculopapular in appearance. Precipitating medications include; chloroquine, ampicillin and isoniazid [7]. Urticarial rash: (hives-red, itchy wheals) and angioedema may be caused by penicillin and irritant contact dermatitis from a number of medications [8].

Pruritus is usually a centrally-mediated reaction; e. g. side effects of opioids and tramadol, which cannot be treated by antihistamines and is usually resolved only by ceasing the medication [8]. Fixed drug eruptions occur where the eruption occurs at the same spot every time that the same medication is administered, e. g. dapsone, metronidazole or aspirin [9].

Acneiform eruptions can be caused by drug-mediated acne eruptions, e. g. anabolic steroids. Psoriasiform eruptions present as worsening of pre-existing psoriasis, e. g., Lithium and $\beta$-blockers. Lichenoid eruptions: resolve by ceasing the drug. They appear as flat mauve lesions, but they can be scaly and may flow into each other, e. g. gold salts, imatinib, interferon- $\alpha[10,11]$. Erythema multiforme is an eruption of target-like lesions and in severe cases the center blisters. Limbs and mucous membranes are involved but not the trunk. It typically occurs two weeks after commencing the drug, e. g: allopurinol and macrolide antibiotics. The condition is reversible on ceasing the medication. Topical corticosteroids can be used for limbs, paracetamol for pain and fever and topical treatment can be used for oral, ocular and genital symptoms $[10,11]$.

Stevens-Johnson syndrome and toxic epidermal necrolysis (SJS/TEN) are the two conditions that may be life-threatening. They are mucocutaneous drug hypersensitivity syndromes where 
blistering and epidermal sloughing occur. SJS causes $<10 \%$ of body surface area skin detachment and TEN causes $>10 \%$ of body surface area skin detachment (e. g., lamotrigine, doxycycline, and cotrimoxazole). Drug withdrawal and supportive treatments include: systemic steroids or ciclosporin, IV immunoglobulin, infection management, thermoregulation, maintenance of fluid balance, skincare and eye care $[10,11]$.

Drug reaction with eosinophilia and systemic symptoms (DRESS) presents as an extensive, inflammatory, maculopapular exanthem with lymph node enlargement, abnormal LFTs and haematological abnormalities, with raised eosinophil count, or presence of atypical lymphocytes. There may also be renal, pulmonary or cardiac involvement (e. g., vancomycin, sulfasalazine, antiretrovirals). It can be fatal $(10 \%)$, and has long latency, characteristically 2-8 w from drug commencement date [12].

Acute generalized exanthematous pustulosisis characterized by widespread monomorphic pustules and fever. Patients are generally unwell and have excessive heat and fluid loss. This condition can be treated by topical potent steroids and can be caused by e. g., lamotrigine, metronidazole, and amoxicillin [13].

Lupus erythematosus is characterized by seroconversion to being antinuclear antibody positive. Patient may recover after drug withdrawal but may retain residual antibodies. The skin eruption is a butterfly-shaped rash on the face, photosensitive erythema on dorsal hands and neck and annular lesions on limbs. It is treated by infliximab and etanercept which targets TNF- $\alpha[10,11]$.

Vasculitis occurs characterized by inflammation of the vessel walls (lower limbs). Drug withdrawal and systemic steroids or IV immunoglobulin can be used in its treatment [14]

To demonstrate the skin side-effects of the most commonly prescribed medications a list of the top 100 [15] used medications in the United Kingdom was identified. The aim of this study was to evaluate the frequency of skin complications and side-effects as a result of the 100 most commonly prescribed medications in the United Kingdom. The dermatology department in New Cross Hospital, (Royal Wolverhampton NHS Trust) aimed to establish a list of medications to give doctors and patients an idea of what medications are capable of producing in the skin as a side-effect.

\section{MATERIALS AND METHODS}

\section{Method}

The list of the top 100 drugs prescribed in the UK was obtained from the Nursing Notes web page (https://nursingnotes.co.uk/the-100most-common-medications-in-uk-hospitals/, 13/02/2019, table 1). Subsequently, the British National Formulary was used to extract the information about the side-effects and was divided and subdivided according to reported frequency. The findings were analysed and represented as histograms.

Table 1: Top 100 most-prescribed medications in UK hospitals (Source: https://nursingnotes. co. uk/the-100-most-common-medicationsin-uk-hospitals/. Accessed on 13/2/2019)

\begin{tabular}{|c|c|c|c|}
\hline Adenosine & Clonazepam & Heparin & Omeprazole \\
\hline Alendronic Acid & Co-amoxiclav & Ibuprofen & Ondansetron \\
\hline Amiloride & Codeine & Indapamide & Oxycodone \\
\hline Amiodarone & Cyclizine & Insulin & Phenobarbitol \\
\hline Amitriptyline & Diazapam & Ipratropium & Phenytoin \\
\hline Amlodipine & Diazepam & Ketamine & Piperacillin/Tazobactam \\
\hline Amoxicillin & Diclofenac & Lansoprazole & Pravastatin \\
\hline Apixaban & Digoxin & Levetiracetam & Prochlorperazine \\
\hline Atenolol & Dihydrocodeine & Levofloxacin & Ramipril \\
\hline Atenolol & Diltiazem & Levomepromazine & Rivaroxaban \\
\hline Atorvastatin & Diltiazem & Levothyroxine & Salbutamol \\
\hline Bendroflumethiazide & Doxazosin & Lisinopril & Sertraline \\
\hline Bisoprolol & Doxycycline & Lorazepam & Simvastatin \\
\hline Bisoprolol & Enoxaparin & Lorazepam & Sodium valproate \\
\hline Bumetanide & Esomeprazole & Losartan & Spironolactone \\
\hline Candesartan & Fentanyl & Meropenem & Temazepam \\
\hline Carbamazepine & Flucloxacillin & Metformin & Theophylline \\
\hline Ceftazidime & Fluoxetine. & Metoclopramide & Tiotropium \\
\hline Cefuroxime & Funderparinex & Midazolam & Tramadol \\
\hline Cephalexin & Furosemide & Mirtazapine & Trazodone \\
\hline Chlordiazepoxide & Gabapentin & Morphine & Trimethoprim \\
\hline Ciprofloxacin & Gabapentin & Naproxen & Vancomycin \\
\hline Citalopram & Gentamycin & Nefopam & Venlafaxine \\
\hline Clarithromycin & Gliclazide & Nifedipine & Warfarin \\
\hline Clindamycin & Haloperidol & Nitrofurantoin & Zopiclone \\
\hline
\end{tabular}

\section{RESULTS AND DISCUSSION}

Cutaneous side effects were reported for all 100 most prescribed medications, but the frequency varied from rare to very common and the severity varied from very mild to very severe.

Rash, pruritus and urticaria were among the most common sideeffects experienced by patients as a result of being prescribed the aforementioned medications (fig. 1).

Other common side-effects in decreasing order were photosensitivity, alopecia, angioedema, SJS, TEN, Jaundice, flushing, dermatitis and acne. These side effects were the most often reported as being side effects but rash and urticaria were the most common skin condition to occur or be present. Adverse drug reactions vary in severity and may be localized to the skin or affect multiple organs
[16]. Hair loss has been associated with the use of 39 out of the 100 most common medications prescribed. Psychotropic drugs can cause hair loss [17] in addition to antidepressants including Selective Serotonin Re-uptake Inhibitors (SSRIs) [18]. Alopecia has also been shown to be associated with the use of valproate on a dosedependent basis [19]. It was noted that upon cessation of the medication, hair growth usually spontaneously returns.

A study conducted in Denmark by the Committee on Adverse Drug Reactions concluded that most common side effects of diuretics, beta-blocking agents, and anti-hypertensive drugs are dermatologically-related [20].

Skin side effects associated with mood stabilizers range from simple rashes to SJS, TEN and exfoliative dermatitis, [21, 22] as appears to be confirmed in this study. 


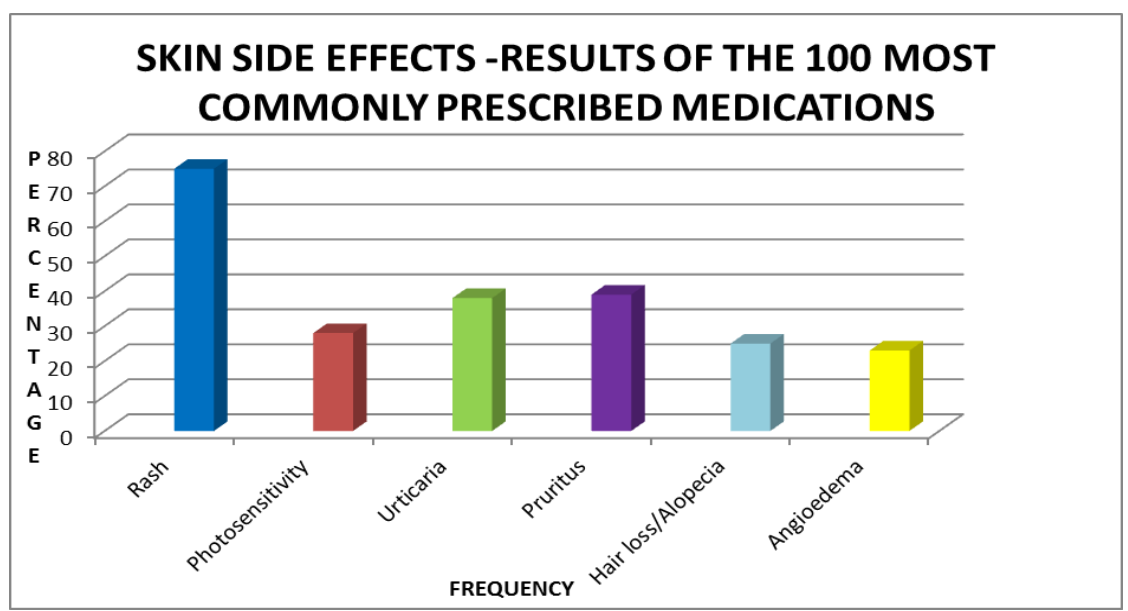

Fig. 1: Most common skin side effects in the top prescribed drugs (more than 10 incidences in 100,000)

Antibiotics and anticonvulsants are two common medication groups causing skin reactions, including maculopapular rashes, urticaria and, in severe cases, SJS, TEN and DRESS [23].

It was found that 28 of the 100 most commonly prescribed medications cause photosensitivity. The photosensitivity most commonly occurs as a phototoxic reaction or less commonly as photoallergic reactions and photo-aggravated dermatitis. The most common drugs causing photosensitivity are nonsteroidal antiinflammatory drugs (NSAIDs), amiodarone, phenothiazines (especially chlorpromazine), retinoids, antibiotics (sulfonamides, tetracyclines, (especially demeclocycline) and quinolones) [23, 24].

SJS and TEN are considered uncommon but serious reactions with high morbidity and mortality. The available data is almost entirely from case reports and observational series. DRESS syndrome is severe and prolonged, affecting multiple organs. To date, no test has been recognised to identify which drug might cause any of these severe reactions especially in patients taking multiple drugs, but when diagnosed, any medication suspected to cause these severe conditions should be promptly withdrawn [2].

Out of the 100 most commonly prescribed medications, $75 \%$ are associated with causing a rash or other dermatological reaction. From these, 18 out of $75(24 \%)$ are listed as 'commonly' or 'very commonly' occurring (fig. 2). This is a high ratio especially taking into account that $38 \%$ cause urticaria 10 out of $38(26 \%)$ common or very common, among less common symptoms which can come under the category of rash/inflammation of the skin like exfoliative dermatitis $9 \%$, acne $4 \%$ and others.

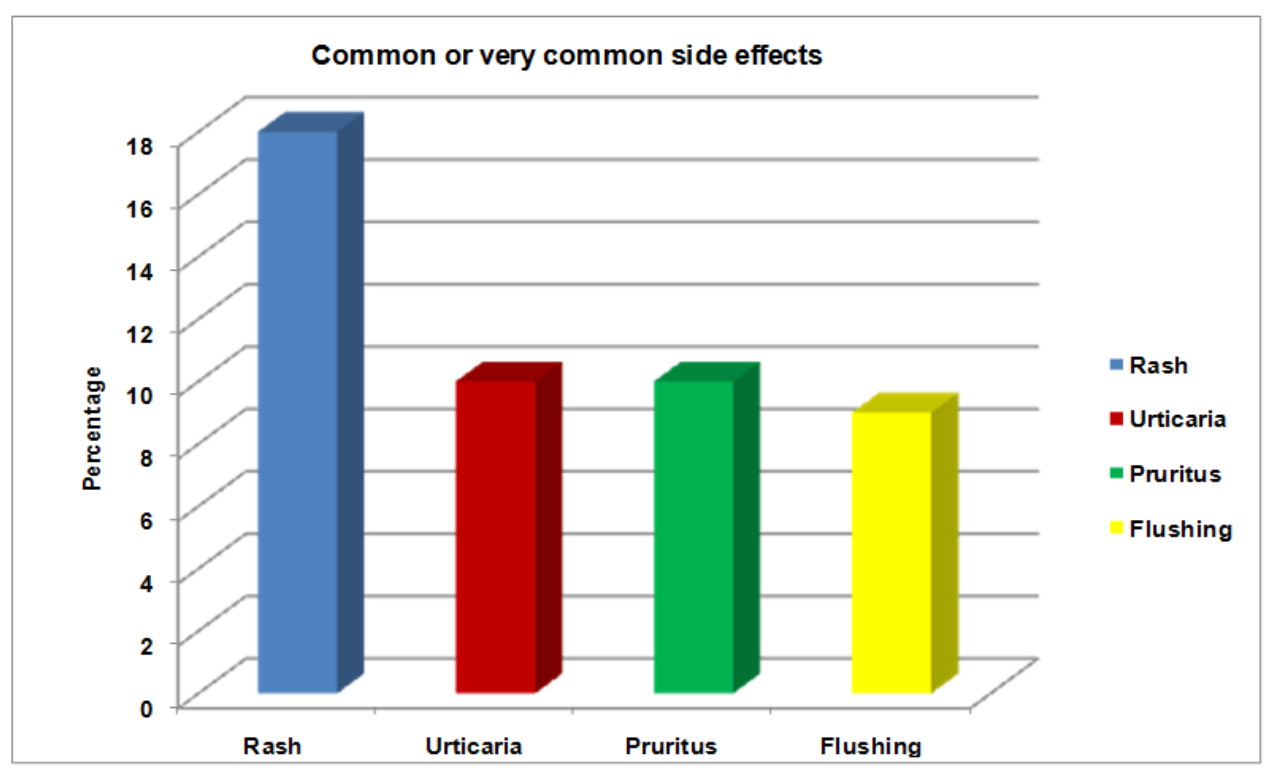

Fig. 2: Common or very common skin side effects in the top prescribed drugs (more than 10 incidences in 100,000)

Angioedema was found in $23 \%$ of all medication with 3 out of 23 (13\%) being common or very common can be combined to the urticarial fig. of $38 \%$ with $(26 \%)$ being common or very common combining the two values $26 \%$ and $13 \%$ (39\%).

The second category was pruritus affecting $39 \%$ of the reported side effects being 10 out of $39(25.6 \%)$ is common or very common. This is significant for an irritating symptom.
The third main category was hair loss, which has medical, cosmetic and social concerns and was reported with about one-quarter of the medications $(25 \%)$ though none in the BNF reported this to be common or very common.

The fourth category was photosensitivity $28 \%$ and flushing $12 \%$ and with 9 out of the 12 were reported as common or very common. If the two fig. are added together, around $40 \%$ of the drugs may cause either photosensitivity or flushing. 
Clearly doctors, pharmacists and most important of all patients need to be aware and prepared of such possible side effects. The International Consensus on drug allergy 25 (Demoly 2014) provides some useful insights into how this agenda may be progressed. They revisited the mechanisms of allergy and the time-lines of the various reactions. From this, they emphasised how crucial the timescale of events can be, to correctly attribute a reaction to a causative agent. Without an accurate time-line the ability to correctly respond to symptoms and arrive at an accurate diagnosis is seriously compromised. Removal of a suspected agent(s) and subsequent rechallenge is possible, but extremely time-consuming and subjects the patient to a double dose of symptoms.

Side effects relating to the skin are common in the 100 most prescribed drugs in our hospitals. The public are not aware of how common these side effects are and health professionals need to be vigilant, and to educate and inform patients on how common these side effects are, and to be able to identify them early. Measures are required to combat severe reactions such as SIS, TEN and DRESS syndrome. Any drug suspected of causing any of these serious conditions should be stopped and immediate treatment commenced.

\section{CONCLUSION}

From this study it is concluded that the best way forward is the education of patients and health professionals to be able to move forward in interpreting skin reactions:

1) When confronted with a common dermatological problem in any patient, always consider the possibility of a drug adverse reaction.

2) To be able to progress with such a conclusion, an accurate timeline is essential; when was each medication started? Exactly when did the reaction first appear? These needs to be identified and clearly recorded at the outset, and this information must be included in any onward referral.

3) Accurate record-keeping relating to suspected drug allergies is essential; it is insufficient to just record the name of a drug as an 'allergy.' Any record of drug sensitivity must contain brief details of what the reaction was; Penicillin-rash; Morphine-hallucinations, at the very least.

4) Reporting suspected drug reactions, even ones believed 'common' to reporting centres will provide more accurate data on the frequency of reactions, the extent of the problem and better inform public policy.

\section{AUTHORS CONTRIBUTIONS}

All the author have contributed equally

\section{CONFLICT OF INTERESTS}

All authors reported no conflict of interest

\section{REFERENCES}

1. Singh RK, Lee KM, Jose MV. The patient's guide to psoriasis treatment. Part 1: UVB Phototherapy. Dermatol Ther (Heidelb) 2016;6:307-1

2. Joint Formulary Committee BNF 74: September 2017. London: Pharmaceutical Press; 2017.

3. Dereure 0. Drug-induced skin pigmentation. Am J Clin Dermatol 2001;2:253-62.

4. Wick JY. Drug-induced nail changes: counting to 10. Pharmacy Times, May 24th Published, Drug-Induced Nail Changes: Counting to 10; 2014. Available from: https:// www.pharmacytimes.com/publications/issue/2014/may2014 /drug-induced-nail-changes-counting-to-10. [Last accessed on 16 Dec 2018]

5. Patel S, Tost A. An overview of the management of druginduced hair and nail disorders. Clin Practice 2014;11:327-40.

6. Coghill AE, Johnson LG, Berg D, Resler AJ, Leca N, Madeleine MM. Immunosuppressive medications and squamous cell skin carcinoma: nested case-control study within the skin cancer after an organ transplant (SCOT) cohort. Am J Transplant 2016;16:565-73.

7. Yawalkar N. Drug-induced exanthems. Toxicology 2005;209:131-4.

8. Lee A. Thomson J Chapter; Drug-induced skin reactions in Le A. (Ed) Adverse Drug Reactions, London, Pharmaceutical Press ISBN 978085369601 8; 2005.

9. Flowers H, Brodell R, Brents M, Wyatt JP. Fixed drug eruptions: presentation, diagnosis, and management. South Med J 2014;107:724-27.

10. Sousa Pinto B, Araujo L, Freitas A, Correia O, Delgado L. Stevens Johnson syndrome/toxic epidermal necrolysis and erythema multiforme drug-related hospitalizations in a national administrative database. Clin Transl Allergy 2018;8:2.

11. French LE. Adverse cutaneous drug eruptions. Chem Immunol Allergy 2012;97:1-235.

12. Choudhary S, McLeod M, Torchia D, Romanelli P. Drug reaction with eosinophilia and systemic symptoms (DRESS) syndrome. J Clin Aesthet Dermatol 2013;6:31-7.

13. Feldmeyer L, Heidemeyer K, Yawalkar N. Acute generalized exanthematous pustulosis: pathogenesis, genetic background, clinical variants and therapy. Int J Mol Sci 2016;17:1214.

14. Radic M, Martinovic Kaliterna D, Radic J. Drug-induced vasculitis: a clinical and pathological review. Neth J Med 2012;70:12-7.

15. Bodell M. Top 100 most prescribed medications in UK Hospitals. Available from: https://nursingnotes.co.uk/wpcontent/uploads/2017/09/Top-100-Most-Prescribed-

Medications-in-UK-Hospitals.pdf [Last accessed on 16 Dec 2018].

16. Ardern Jones M, Aqnd Friedman PS. Skin manifestations of drug allergy. Br J Clin Pharmacol 2011;71(S)672-83.

17. Mermi O, Atmaca M, Kilic F, Gurok MG, Kuloghi M. Fluoxetine induced hair loss, a case report', dusunen ADAM. J Psychiatry Neurol Sci 2014;27:266-9.

18. Thomson SR, Mamulpet V, Adiga S. Sodium valproate induced alopecia: a case series. J Clin Diagnostic Res 2017;11:1-2.

19. Thestrup Pederson K. Adverse reactions in the skin from antihypertensive drugs. Damish Med Bull 1987;34 Suppl 1:3-5.

20. Rebok F, Perez Roldan ML, Daray FM. Cutaneous side effects due to psychiatric drugs. Vertex (Beunos Aries, Argentina) 2008;19:348-56.

21. Suastegui Rodrigues L, Campos Jimenez KI, Dominguez Cherit I, Mendez Flores S. Adverse cutaneous reactions to drugs. Revista Medica Del Instituto Mexicano Del Seguro Social 2018;S6:64-70.

22. Lugovic Mihic L, Duvancic T, Fercek L, Vukovic P, Japundżic I, Cesic D. Drug induced photosensitivity-a continuing diagnostic challenge. Acta Clin Croatia 2017;56:277-83.

23. Zuba EB, Koronowska S, Osmola Mankowska A, Jenerowicz D. Drug induced photosensitivity. Acta Dermatovenerologica Croatia ADC 2016;24:55-64.

24. Alvarado SA, Munoz Mendoza D, Bahna SL. High-risk drug rashes. Ann Allergy Asthma Immunol: Official Publication Am College Allergy, Asthma, Immunol 2018;121:552-60.

25. Demoly P, Adkinson NF, Brockow K, Castells M, Chiriac AM, Greenberger PA, et al. International consensus on drug allergy. Allergy 2014;69:420-37. 